\title{
Measurement of myocardial blood flow response to the cold pressor test with myocardial perfusion CMR
}

\author{
Timothy A Fairbairn*, Adam Mather, Adbul Larghat, John Greenwood, Sven Plein \\ From 2011 SCMR/Euro CMR Joint Scientific Sessions \\ Nice, France. 3-6 February 2011
}

\section{Objective}

To measure Cold Pressor Test (CPT)-induced changes in $\mathrm{MBF}$ and compare them to adenosine induced (endothelial-independent) changes in MBF.

\section{Background}

The CPT induces endothelial-dependent vasodilation with increased myocardial blood flow (MBF) in normal coronary arteries. It has been used to demonstrate abnormal coronary vasomotion by invasive (angiography) and non-invasive techniques (SPECT and PET). Cardiovascular Magnetic Resonance (CMR) allows the assessment of MBF in separate myocardial layers due to its high spatial resolution, but has not previously been used to measure physiological responses to CPT.

\section{Methods}

Eleven healthy volunteers (age $23 \pm 5.4,64 \%$ male) attended for a CMR perfusion scan (Phillips Intera 1.5T, $0.05 \mathrm{mmol} /$

$\mathrm{kg}$ Gd-DTPA, spatial resolution $2.3 \times 2.3 \mathrm{~mm}$ ), performed at rest, during CPT (120s of foot immersion in $0-4^{\circ} \mathrm{C}$ water) and adenosine hyperaemia (140 $\mathrm{mcg} / \mathrm{kg} / \mathrm{min}$ for 4 minutes). Each perfusion scan was separated by 15 minutes. Heart rate (HR) and blood pressure (BP) were simultaneously recorded This information was then used to calculate mean arterial pressure $(\mathrm{MAP}=2 *$ Diastolic $\mathrm{BP}(\mathrm{DBP})+$ Systolic BP $(\mathrm{SBP}) / 3)$, rate-pressure product $(\mathrm{RPP}=\mathrm{HR} \mathrm{x}$ $\mathrm{SBP})$ and coronary vascular resistance $(\mathrm{CVR}=\mathrm{MAP} / \mathrm{MBF})$. $\mathrm{MBF}(\mathrm{ml} / \mathrm{g} / \mathrm{min})$ was estimated for a mid-ventricular slice by Fermi-constrained deconvolution.

\section{Results}

MBF increased significantly from rest to CPT $(1.5 \pm 0.5$ to $2.3 \pm 0.6 \mathrm{ml} / \mathrm{g} / \mathrm{min}, \mathrm{p}=0.004)$ and from CPT to stress $(4.4 \pm 0.8, \mathrm{p}<0.001)$ (Table 1). Endocardial MBF was significantly higher than epicardial MBF at rest $(\mathrm{p}<0.001)$ and during CPT $(\mathrm{p}=0.008)$, however during adenosine hyperaemia epicardial MBF was higher $(\mathrm{p}=0.043)$.

Table 1 Haemodynamic parameters and Myocardial Blood Flow ( $\mathrm{ml} / \mathrm{g} / \mathrm{min})$, measured at rest, peak cold pressor test (CPT) and adenosine stress

\begin{tabular}{|c|c|c|c|c|c|}
\hline & REST & $P$ value & CPT & $P$ value & ADENOSINE \\
\hline Heart Rate (bpm) & $70 \pm 9$ & 0.001 & $84 \pm 12$ & 0.02 & $97 \pm 12$ \\
\hline Systolic Blood Pressure (mmHg) & $120 \pm 10$ & 0.025 & $132 \pm 16$ & 0.002 & $114 \pm 14$ \\
\hline Diastolic Blood Pressure (mmHg) & $66 \pm 7$ & 0.001 & $78 \pm 9$ & $<0.001$ & $60 \pm 9$ \\
\hline Mean Arterial Pressure $(\mathrm{mmHg})$ & $84 \pm 6$ & 0.022 & $94 \pm 15$ & $<0.001$ & $78 \pm 10$ \\
\hline Rate Pressure Product $(\mathrm{mmHg} / \mathrm{min})$ & $8441 \pm 1198$ & $<0.001$ & $11157 \pm 2018$ & 0.972 & $11184 \pm 2031$ \\
\hline Coronary Vascular Resistance $(\mathrm{mmHg} / \mathrm{ml} \operatorname{min~} \mathrm{g})$ & $60.5 \pm 14.8$ & 0.01 & $43.9 \pm 15.4$ & $<0.001$ & $18.5 \pm 4.2$ \\
\hline Myocardial Blood Flow (ml/g/min) & $1.48 \pm 0.15$ & 0.004 & $2.31 \pm 0.18$ & $<0.001$ & $4.36 \pm 0.25$ \\
\hline Endocardial Blood Flow (ml/g/min) & $1.7 \pm 0.6$ & NA & $2.38 \pm 0.5$ & NA & $4.09 \pm 0.7$ \\
\hline Epicardial Blood Flow (ml/g/min) & $1.38 \pm 0.5$ & NA & $2.09 \pm 0.7$ & NA & $4.53 \pm 1.1$ \\
\hline
\end{tabular}

University of Leeds, Leeds, UK 


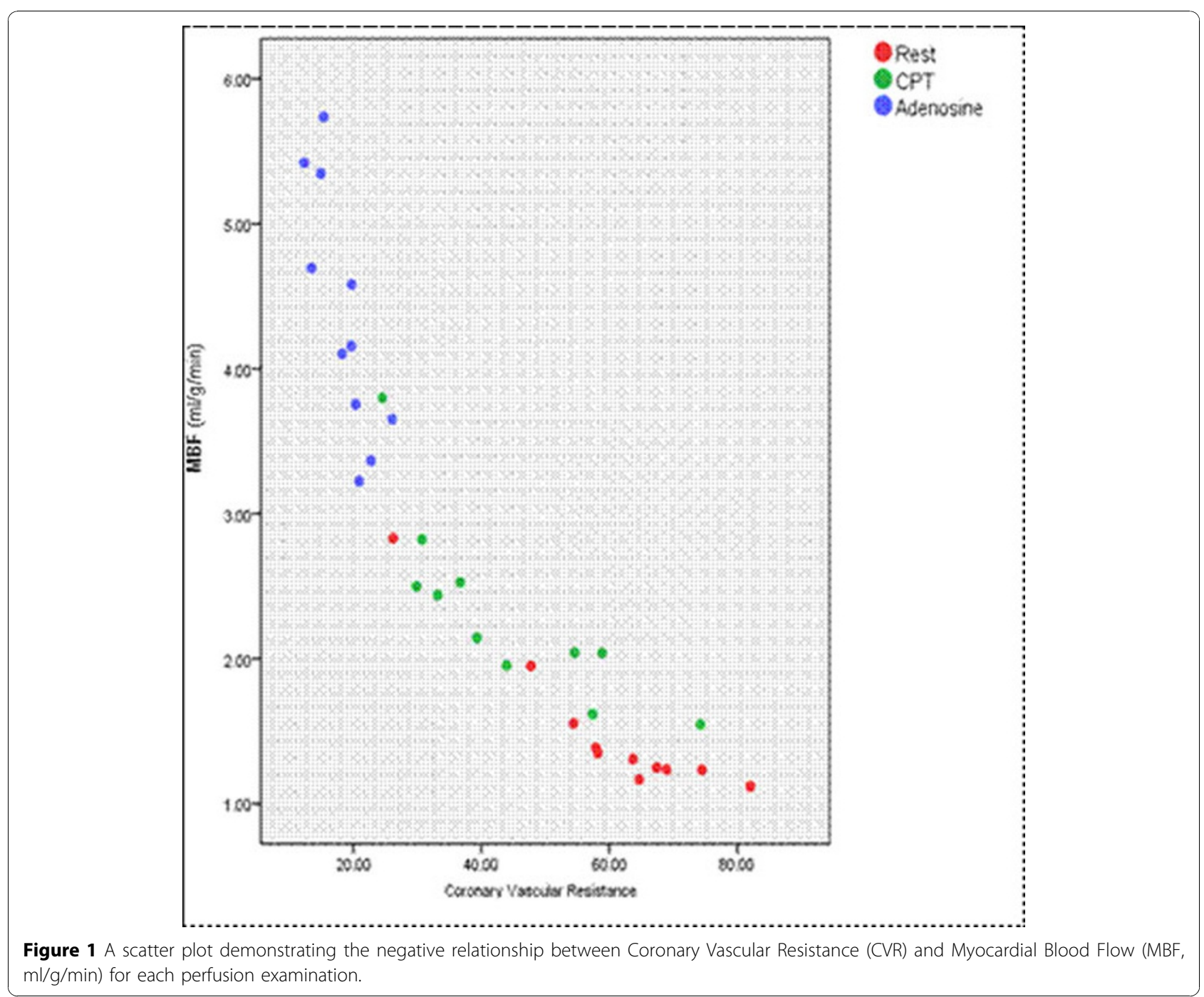

Regression analysis of haemodynamic factors identified Coronary Vascular Resistance (CVR) as the only independent predictor of MBF during rest, $\mathrm{CPT}$ and adenosine, (Figure 1).

\section{Discussion}

Perfusion-CMR permits assessment of endothelialdependent $(\mathrm{CPT})$ and endothelial-independent (adenosine) MBF in a single examination. Furthermore, CMR demonstrates differences in the physiological response to CPT and maximal hyperaemia between the endocardium and epicardium. Future studies should establish the role of this new method in at risk groups such as those with diabetes or smokers.

Published: 2 February 2011
doi:10.1186/1532-429X-13-S1-P312

Cite this article as: Fairbairn et al:: Measurement of myocardial blood flow response to the cold pressor test with myocardial perfusion CMR. Journal of Cardiovascular Magnetic Resonance 2011 13(Suppl 1):P312.

\section{Submit your next manuscript to BioMed Central} and take full advantage of:

- Convenient online submission

- Thorough peer review

- No space constraints or color figure charges

- Immediate publication on acceptance

- Inclusion in PubMed, CAS, Scopus and Google Scholar

- Research which is freely available for redistribution 